CONFERENCIA

\title{
¿ESTÁ OBSOLETA LA FÍSICA DE ARISTÓTELES?*
}

\begin{abstract}
Alejandro G. Vigo
El presente trabajo ofrece una interpretación de conjunto del modelo teórico presentado por Aristóteles en su Física, con referencia a su peculiar diseño temático y metódico. El abordaje a los objetos y procesos naturales practicado por Aristóteles es caracterizado como un acceso fenomenológico, por oposición a lo que sería un enfoque predominantemente causal-reductivo. Dicho acceso fenomenológico apunta, en lo fundamental, a una dimensión de manifestación y experiencia que es previa a toda distinción tajante entre lo que moder-
\end{abstract}

Alejandro G. Vigo. Doctor en Filosofía por la Universidad de Heidelberg (Alemania). Profesor Adjunto Ordinario, Instituto de Filosofía, Pontificia Universidad Católica de Chile, Santiago de Chile.

* Dos versiones previas de este texto, más amplias y de carácter más técnico, han servido como base para conferencias dictadas en el marco de congresos dedicados a la discusión de diferentes aspectos del pensamiento de Aristóteles, y han sido publicadas con el título "Indiferentismo Ontológico y Fenomenología en la Física de Aristóteles” en revistas especializadas. La presente versión corresponde a la exposición realizada el 22 de junio de 2006 en el marco del ciclo de conferencias “Aristóteles, filósofo para este tiempo”, organizado por el Centro de Estudios Públicos (Santiago de Chile). El texto fue reducido en extensión y despojado, en lo posible, de los aspectos más técnicos, a fin de adaptarlo a los requerimientos de un ciclo destinado a público no especializado. Las traducciones de los textos griegos citados pertenecen al autor de este trabajo.

Véanse también en esta edición las conferencias dictadas por Óscar Godoy y Alfonso Gómez-Lobo durante el ciclo “Aristóteles, filósofo para este tiempo” (N. del E.)

Estudios Públicos, 102 (otoño 2006). 
namente se denomina el 'sujeto' y el 'objeto' del conocimiento. Sobre esta base, se defiende la tesis de que la obsolescencia de la mayor parte de aquellos aspectos que pertenecerían a lo que hoy se denomina la ciencia empírica de la naturaleza no acarrea, de suyo, una paralela obsolescencia de la concepción aristotélica, en el plano correspondiente a la reflexión filosófica. Por el contrario, leída fundamentalmente como una fenomenología de la experiencia de la naturaleza, situada metódicamente en el nivel descriptivo que corresponde al acceso propio de la actitud pre-reflexiva, la concepción elaborada por Aristóteles posee, desde el punto de vista estrictamente filosófico, todavía hoy una notable capacidad de interpelación y una sorprendente actualidad.

\section{Introducción}

Pocs

ocas obras de Aristóteles han tenido un destino tan azaroso en la historia de su recepción como la Física: tras un largo período de escasa o ninguna repercusión por quedar sumida en el olvido, el modelo teórico expuesto en ella pasó, de un modo casi repentino en comparación con la prolongada latencia precedente, a formar parte fundamental del canon científico occidental en el Medioevo tardío y los comienzos de la Modernidad, para luego, con el advenimiento de la ciencia matemática de la naturaleza, ir cayendo progresivamente en el descrédito, al punto de llegar a ser considerado con mucha frecuencia, incluso hasta el día de hoy, como un ejemplo paradigmático del tipo de desviaciones y arbitrariedades a las que puede conducir lo que sería un enfoque metódicamente inadecuado, de carácter ingenuo y especulativo, en campos que deben quedar reservados a la competencia de la ciencia empírica y preservados así de la mera especulación filosófica.

Es todavía frecuente toparse con un característico meta-relato que, en el marco del intento de reconstrucción del proceso histórico que lleva al triunfo final de la ciencia moderna y la civilización tecnológica basada en ella, presenta a la especulación filosófica sobre la naturaleza de autores como Platón y Aristóteles como directamente responsable de haber sofocado el impulso científico naciente de los primeros cosmólogos presocráticos y de dar así paso a un tipo de abordaje filosófico-especulativo, en el sentido peyorativo del término, que habría significado en la práctica un retraso de más de mil años en el progreso del conocimiento científico de la 
naturaleza: la ciencia moderna de la naturaleza, con su método empírico e hipotético, no habría hecho, por su parte, sino retornar a la mejor tradición del racionalismo naturalista jonio y radicalizar tendencias presentes ya en ella ${ }^{1}$. En círculos vinculados con la tarea de divulgación científica, de filiación más o menos marcadamente cientificista, esta versión del desarrollo de la historia de la ciencia ha llegado a ser incluso un lugar común. En una época marcada decisivamente por el fenómeno del auge de la ciencia y la tecnología basada en ella, el descrédito así sancionado de la filosofía natural aristotélica hizo sentir claramente su influencia incluso en el ámbito, mucho más reducido y mucho menos popular, de la investigación especializada del pensamiento de Aristóteles. En tal sentido, el autor de un libro ya clásico sobre la Física, aparecido a comienzos de los años sesenta y causante directo en buena medida del resurgimiento actual del interés por la obra, pudo decir que, frente a la mucha atención dedicada por los especialistas a las otras obras mayores de Aristóteles, la Física podía considerarse todavía en esos años como terra incognita ${ }^{2}$.

No deja de ser sorprendente y, a la vez, aleccionador el hecho de que tanto el transitorio éxito, en la tardía Edad Media, de la concepción expuesta por Aristóteles en su Física como su posterior descrédito, con el advenimiento del Renacimiento y la Modernidad, respondan ambos, en buena medida, a una similar interpretación de los objetivos y el alcance de dicho modelo teórico. Se trata de una interpretación que sobreexige irremediablemente a la concepción aristotélica, en la medida en que le reclama las funciones explicativas propias de lo que debería ser una teoría unificada que dé cuenta del comportamiento de la realidad física en su conjunto, desde el punto de vista propio de una ciencia de la naturaleza. Es verdad que esta sobreexigencia interpretativa procede en uno y otro caso de una constelación básica fundamentalmente opuesta: en la interpretación medieval, la ausencia de una nítida distinción entre la vieja filosofía natural y lo que hoy llamamos la ciencia empírica de la naturaleza; en el caso de la tradición moderna, inversamente, la cada vez más decidida separación entre ambas, a partir de la constitución de la física como ciencia independiente, con el consiguiente descrédito de la filosofía natural escolástica, privada ahora de su objeto propio y su área específica de competencia, y condenada así a la obsolescencia.

No es menos cierto, sin embargo, que ambos enfoques resultan convergentes, en la medida en que no permiten dar cuenta adecuadamente de la

\footnotetext{
${ }^{1}$ Cf. Popper (1963); véase esp. pp. 136, 151.

2 Véase Wieland (1970), p. 15; véase también Wieland (1960-61), p. 206.
} 
especificidad del modo aristotélico de abordaje de la naturaleza, por oposición al característico de una ciencia empírica, en el sentido actual del término. En efecto, hay fuertes razones para sostener que, en su finalidad explicativa básica y de acuerdo con su diseño metódico específico, el modelo teórico presentado por Aristóteles en su Física no constituye tanto una teoría empírica de la naturaleza en el sentido habitual, cuanto más bien el bosquejo de una peculiar ontología del ente natural, en cuanto éste se caracteriza esencialmente por estar sujeto a movimiento o cambio. Dicha ontología no sólo se caracteriza por una peculiar demarcación de su ámbito temático específico, sino también - y de modo no menos esencial— por una determinada concepción metódica. Ésta puede denominarse con justicia como fenomenológica, y apunta a un ámbito de experiencia situado más allá o, si se prefiere, más acá de la distinción habitual entre lo que modernamente se suele denominar el ‘sujeto’ y el ‘objeto' del conocimiento. Como intentaré mostrar a continuación, es justamente en el ámbito correspondiente a los análisis estructurales de determinados fenómenos básicos, vinculados con la experiencia inmediata de la naturaleza y el movimiento natural, donde la concepción de Aristóteles presenta elementos que le conceden todavía una sorprendente actualidad y relevancia desde el punto de vista filosófico, independientemente del hecho de que el modelo explicativo que Aristóteles presenta ya no esté en condiciones de calificar como una teoría científica de la naturaleza, en el sentido actual del término.

\section{La filosofía natural como ontología del ente sujeto a movimiento y su carácter fenomenológico}

He dicho que la Física de Aristóteles no presenta tanto lo que actualmente llamaríamos una ‘teoría física', aunque de corte más primitivo que las que hoy sirven de base a la tarea de los científicos de la naturaleza, sino, más bien, una determinada ontología del ente natural, en cuanto ente sujeto a movimiento, caracterizada por una peculiar concepción metódica. Con esto no quiero dar a entender que Aristóteles haya operado de modo expreso con vistas a dicho contraste entre ambos modos de abordar el estudio de la naturaleza. Lo que la tesis que he formulado quiere significar es algo mucho más modesto, a saber: que la hipótesis hermenéutica más productiva a la hora de dar cuenta de la especificidad del abordaje a los fenómenos naturales practicado por Aristóteles en su Física consiste en leer la obra desde la perspectiva abierta por la idea de una ontología del ente 
natural, en cuanto esencialmente caracterizado por el hecho del movimiento ${ }^{3}$.

Por cierto, no faltan en la obra pasajes que discuten problemas específicos que desde la Modernidad pertenecen, sin duda, a la esfera de competencia propia de la ciencia empírica de la naturaleza. Se trata justamente de aquellos pasajes a los que se suele apelar a la hora de ilustrar la obsolescencia de los modelos explicativos propuestos por Aristóteles para dar cuenta del comportamiento de los entes de la realidad física. Piénsese, para poner un ejemplo paradigmático, en el famoso tratamiento aristotélico del movimiento de los proyectiles, sobre la base de la asunción de la necesidad de contacto entre el motor y lo movido (cf. Fís. IV 8, 215a14-19; VII 2; VIII 10, 266b27-267a20). Ya Filópono sometió este modelo explicativo a una crítica devastadora, la cual jugó un papel importante en la motivación de la posterior caída en descrédito de la teoría aristotélica del movimiento. Pero esto en nada altera el hecho de que, desde el comienzo mismo de la indagación desarrollada en la Física, Aristóteles pretende situarse fundamentalmente en la perspectiva que abre la pregunta por los principios (archaî) que dan cuenta de la constitución ontológica del ente natural sujeto a movimiento, en tanto sujeto a movimiento (cf. I 1, 184a10-16). La naturaleza constituye, como tal, un principio de movimiento (y reposo) en los entes naturales (cf. II 1, 192b20-27). Por lo mismo, el fenómeno del movimiento debe ocupar el centro de una indagación referida a dichos principios.

Importante desde el punto de vista del método es el hecho de que la cuestión de la existencia del movimiento queda, desde un comienzo, excluida de dicha indagación acerca de los principios del ente natural en tanto móvil. Que hay movimiento es, para Aristóteles, un hecho de experiencia (cf. I 2, 185a13 s.), del cual la filosofía natural debe partir como de un presupuesto: su puesta en discusión no aporta nada a la problemática propia de la filosofía de la naturaleza (cf. I 2, 184b25-185a20). Se trata, pues, de indagar no si el movimiento es, sino, más bien, qué es (cf. III 1, 200b12-15). El principio metódico que sanciona la puesta entre paréntesis de la pregunta por la existencia del movimiento supone un distanciamiento respecto de

${ }^{3}$ Sobre este punto ha insistido M. Heidegger. Véase Heidegger (1939), esp. pp. 312 y ss. Intérpretes vinculados estrechamente con el pensamiento de Heidegger, tales como W. Bröcker y P. Aubenque, han desarrollado en detalle esta línea interpretativa. Así, Bröcker (1974) ha presentado una reconstrucción de conjunto del pensamiento de Aristóteles que enfatiza el papel central que desempeña en él la noción de movimiento. Por su parte, Aubenque (1962), esp. pp. 412-484, ha puesto de manifiesto la conexión de la concepción de la física como una ontología del ente en movimiento con el contenido nuclear de la teoría aristotélica de las categorías y con el problema de la posibilidad de la ciencia del ser. 
todo abordaje puramente especulativo al ámbito de los fenómenos naturales. Pero no menos cierto es, sin embargo, que la orientación básica a partir de la pregunta por la esencia del movimiento y por los principios constitutivos del ente sujeto a movimiento como tal sitúa a la indagación aristotélica, desde un comienzo, en un plano que queda de antemano sustraído a la esfera de competencia propia de una ciencia empírica de la naturaleza. En virtud de su orientación básica, la filosofía natural de Aristóteles se interna, pues, por un camino intermedio entre la metafísica especulativa, por un lado, y la ciencia empírica de la naturaleza, por el otro.

Ahora bien, es importante advertir que el análisis del movimiento y el ente natural sujeto a movimiento llevado a cabo por Aristóteles en Física se sitúa en un nivel de descripción que guarda correspondencia directa no sólo con las premisas fundamentales de la ontología aristotélica, sino también con los mecanismos de identificación de entidades y con las asunciones ontológicas elementales que subyacen al acceso pre-filosófico e incluso pre-teórico al mundo y la naturaleza, en la experiencia inmediata y la praxis cotidiana. En efecto, el mundo tematizado por la física aristotélica es fundamentalmente un mundo de cosas subsistentes dotadas de determinadas propiedades, vinculadas relacionalmente con otras cosas subsistentes, y sujetas a diferentes tipos de procesos de movimiento o cambio.

Esta decisión de base se corresponde con el esquema ontológico presentado en obras como Metafísica y, de otro modo, también Categorías, el cual está basado en la distinción categorial entre el objeto (sustancia) y sus determinaciones (accidentes). Justamente en conexión con esta distinción básica se pone de manifiesto en Física una tendencia a la desustancialización de entidades que la tradición filosófica había concebido, al menos implícitamente, en términos sustancialistas o cuasi-sustancialistas. Dicha tendencia, que testimonia claramente acerca de la madurez filosófica alcanzada por Aristóteles ya en Física, adquiere expresión, sobre todo, en el conjunto de breves tratados contenidos en los libros III y IV, en los cuales Aristóteles analiza, como se dijo, el movimiento, el infinito, el lugar, el vacío y el tiempo. Todas estas reales o — como en el caso del vacío, a juicio de Aristóteles - supuestas entidades habían sido elevadas por diferentes pensadores en la tradición filosófica precedente a la categoría de principios, a los que correspondería un tipo de existencia que, en la terminología propia de la filosofía aristotélica, debería designarse como sustancial o cuasi-sustancial. En conexión con dicha tendencia a la desustancialización Aristóteles concede a las mencionadas entidades un estatuto ontológico mucho más modesto: no las concibe como cosas dotadas de existencia sustancial, sino, más bien, como propiedades o determinaciones de los objetos sustan- 
ciales o bien de los procesos a los que dichos objetos sustanciales quedan sometidos. Esta tendencia a la desustancialización de entidades a las que la tradición había estado inclinada a concebir en términos sustanciales o cuasi-sustanciales no resulta en absoluto incompatible con el sustancialismo aristotélico. Por el contrario, la actitud más bien restrictiva a la hora de admitir entidades que merezcan el título de 'objetos', en el sentido más propio del término, no constituye sino el reverso de la concepción ontológica según la cual los objetos sustanciales han de verse como aquellas entidades básicas de cuya existencia depende, de modo directo o indirecto, la existencia de todo lo demás.

Pues bien, la decisión inicial básica que vincula a la filosofía natural con el nivel de descripción que corresponde a la experiencia inmediata de la naturaleza, tal como ésta acontece ya en el acceso pre-teórico al mundo y la praxis cotidiana, está muy lejos de constituir, como podría parecer a primera vista, y como de hecho se ha sostenido a veces, un punto de partida dogmático, que bloquea de antemano la posibilidad de elaborar modelos explicativos tal vez contraintuitivos, pero, a la vez, más eficaces a la hora de dar cuenta del comportamiento de los entes naturales, desde el punto de vista de una ciencia empírica de la naturaleza. Por el contrario, en el caso de Aristóteles, tal decisión inicial debe verse, más bien, como el resultado de una toma de posición consciente con relación a la pregunta por el objeto específico al que debe apuntar una indagación filosófica de la naturaleza. Esto se advierte no sólo a partir del modo en que Aristóteles caracteriza el objeto y el alcance de la investigación llevada a cabo en Física, sino también, y con especial nitidez, a partir de su actitud crítica frente a las concepciones elaboradas por algunos de los principales representantes de la tradición presocrática. Aquí hay que mencionar, sobre todo, la clara toma de distancia de Aristóteles respecto de modelos explicativos que, como el atomismo de Leucipo y Demócrito, intentan dar cuenta de los fenómenos observables, concibiéndolos como meros epifenómenos de cosas y procesos que tienen lugar en el nivel de una micro-estructura que queda, como tal, sustraída al acceso inmediato a través de la experiencia sensible. Esta orientación metódica básica de corte causal-reductivo queda expresada de modo ejemplar en la famosa sentencia de Demócrito: "por convención dulce, por convención amargo, por convención caliente, por convención frío, por convención color; en realidad, átomos y vacío" (68B9 DK). Nuestra fuente para este fragmento, el escéptico Sexto Empírico (Adv. Math. VII 135), lo cita para ilustrar la tesis de que no aprehendemos en verdad nada que sea objetivamente originario, sino tan sólo lo que se aparece a través de las modificaciones de nuestro cuerpo, de modo que no captaríamos nada tal como se manifiesta en verdad, sino sólo tal como se nos aparece. A juicio de Sexto, 
esta tesis implicaría la supresión lisa y llana del campo fenoménico abierto por la experiencia sensible.

Con su punto de partida metódico, que busca orientación básicamente a partir de la distinción categorial entre el objeto y sus determinaciones, Aristóteles se distancia claramente de este tipo de abordaje teórico, caracterizado por el recurso a modelos explicativos dotados de un fuerte componente reduccionista de corte causal. Esto no impide, por cierto, que el propio Aristóteles pueda recurrir en su filosofía natural a otros modelos de explicación causal y a otras estrategias de explicación reductiva, como efectivamente lo hace. Pero lo característico de tales intentos explicativos está dado justamente por el hecho de que el esfuerzo por remontarse al plano de los principios que dan cuenta de los fenómenos no toma habitualmente en ellos la forma de una reducción causal de la macro-estructura a una micro-estructura sustraída al acceso perceptivo, en un ir más allá de lo perceptivamente dado, que corre el riesgo de perder finalmente contacto con su propio punto de partida. El intento de explicación acontece aquí, más bien, en la forma de un retroceso, por vía de tematización reflexiva, hacia aquello que está siempre ya presupuesto en todo acceso pre-teórico al mundo abierto a través de la experiencia sensible y en todo modo de referirnos significativamente a él por medio del lenguaje. Como lo muestra ejemplarmente el análisis de la estructura básica del cambio en Física I 7, por medio de la famosa tríada de principios sustrato-forma-privación, Aristóteles se orienta metódicamente a partir de un peculiar presupuesto de congruencia entre lo que en cada caso ha de ser explicado y aquello a lo que se apela para explicarlo, entre el explanandum y su correspondiente explanans: los principios aristotélicos son siempre principios de cosas, y quedan, como tales, referidos de modo inmediato a aquello de lo que dan cuenta. Pero las “cosas” de las que se pretende dar cuenta por medio de dichos principios no son entidades u objetos cualesquiera, sino más bien aquellos que se nos ofrecen de modo directo en el acceso inmediato al mundo de la experiencia. Además, se intenta dar cuenta de ellos no bajo cualquier punto de vista posible, sino, más bien, con arreglo a las características con que se nos ofrecen en dicho acceso inmediato y dentro de los límites propios de tal acceso. Esto explica en buena medida, a mi juicio, por qué la concepción presentada por Aristóteles en Física ha podido verse como un ejemplo paradigmático de un modelo teórico en el cual el análisis fenomenológicodescriptivo juega un papel mucho más importante que la explicación de corte causal-reductivo ${ }^{4}$.

${ }^{4}$ A este respecto véanse las excelentes observaciones de Wieland (1970), pp. 335 y ss. 
Todo lo señalado apunta a poner de relieve un hecho básico que, a mi juicio, no siempre ha sido considerado en su verdadero alcance, a la hora de dar cuenta del diseño temático y metódico del modelo teórico presentado por Aristóteles en Física, a saber: en la medida en que se sitúa desde el comienzo en el nivel de descripción correspondiente a la experiencia inmediata de la naturaleza a través del acceso pre-reflexivo y pre-teórico al mundo circundante, la concepción aristotélica apunta, de hecho, a una dimensión de indiferenciación situada, por así decir, más acá de la separación tajante entre el 'sujeto' y el 'objeto' de dicha experiencia, al menos tal como ésta se concibe habitualmente, sobre todo, bajo la influencia de la temática propia de la filosofía de la conciencia, característica de la Modernidad. En lo que sigue mostraré brevemente, a partir del examen de unos pocos ejemplos especialmente escogidos, cómo algunas de las estructuras fundamentales tematizadas por Aristóteles en la Física se caracterizan precisamente por su pertenencia a tal dimensión de indiferenciación. Y trataré de mostrar que dicho carácter de indiferenciación, en el sentido particular indicado, está ontológicamente fundado, en la medida en que se apoya en determinados rasgos estructurales de la concepción aristotélica en torno al tipo de actualidad que es propio de los objetos sensibles y, de modo más general, en torno a las relaciones entre el alma y el mundo o la naturaleza.

\section{Indiferentismo ontológico en el análisis fenomenológico de la naturaleza}

1) Un primer ejemplo relevante para la ilustración del carácter indiferentista de las estructuras tematizadas por Aristóteles en Física viene dado por el famoso y discutidísimo pasaje del tratado del tiempo de IV 10-14, en el cual Aristóteles establece expresamente una correlación estructural entre el tiempo como número del movimiento y el alma como instancia capaz de llevar a cabo el acto de numeración. Se trata concretamente del pasaje 223a21-29. Aristóteles plantea allí la pregunta referida a la relación entre tiempo y alma (223a21 s.), y ofrece una respuesta muy matizada. Dicha respuesta puede, sin embargo, parecer sorprendente, cuando se parte de ciertas ideas preconcebidas acerca de lo que debería ser la posición de un pensador como Aristóteles con referencia a la alternativa, supuestamente excluyente, entre posiciones de corte 'realista' y concepciones de corte 'idealista'. El texto reza como sigue:

Por otra parte, podría plantearse la cuestión de si en caso de no existir el alma habría o no tiempo. Pues si es imposible que 
exista aquello que ha de llevar a cabo la numeración, también será imposible que haya algo numerable, de modo que tampoco habrá número, pues número es o bien lo numerado o bien lo numerable. Y si ninguna otra cosa es por naturaleza capaz de numerar sino el alma y el intelecto del alma, 'entonces' es imposible que haya tiempo en caso de no haber alma, a no ser como aquello siendo lo cual es el tiempo, v. gr. si es posible que haya movimiento sin alma. Pues lo anterior y posterior es en el movimiento, y tiempo son estas “determinaciones" en cuanto numerables. (Fís. IV 14, 223a21-29)

El pasaje ha dado lugar desde la antigüedad a las más diversas interpretaciones, en un arco que va desde una lectura radicalmente realista a una radicalmente idealista ${ }^{5}$, y puede decirse que las dificultades que ha planteado tradicionalmente tienen que ver, en buena medida, justamente con el hecho de que la posición de Aristóteles no se deja clasificar, sin más, bajo ninguno de los dos miembros de la alternativa 'realismo’-‘idealismo'.

La respuesta de Aristóteles, que se basa en la previa caracterización del tiempo como número del movimiento (cf. IV 11, 219a1-2), establece claramente una correlación estructural entre el tiempo y el alma: si es imposible la existencia de algo que lleve a cabo la numeración, también será imposible la existencia de algo numerable, con la consecuencia de que tampoco habrá número, ya que número es o bien lo que ha sido numerado o bien lo que puede numerarse (cf. 223a22-25). En términos de la vinculación tiempo-alma tenemos las siguientes correlaciones: por un lado, 'lo que ha de llevar a cabo la numeración’ o ‘lo que es por naturaleza capaz de numerar’ equivale al alma o, más precisamente, al intelecto (noûs) del alma; por otro lado, el número, en el sentido de lo numerado o lo numerable, equivale al tiempo. Por lo tanto, la tesis 'no hay número si no hay un correspondiente agente de numeración’ equivale aquí a la tesis ‘no hay tiempo sin alma’ (223a25-26). En esta argumentación hay que destacar varios aspectos.

a) En primer lugar, Aristóteles no parece estar afirmando simplemente que en un mundo donde nada pueda numerar o contar nada puede ser numerado o contado. El punto parece ser, más bien, que en un mundo semejante no habría tampoco unidades de numeración o cuenta, es decir, lo que Aristóteles llama en otro contexto el 'número por medio del cual numeramos’ (cf. IV 11, 219b5-8). Hay que tener en cuenta, en este sentido, que ya para la determinación de los lapsos o segmentos temporales que serán empleados como unidades de cuenta o medida en la medición de un tiempo

${ }^{5}$ Para un esbozo de la historia de la interpretación del pasaje, véase Volpi (1988), esp. pp. 28-53. 
total se requiere la intervención de un agente de numeración (v. gr. el alma), que determine las diferentes fases en un movimiento por medio de la aplicación sucesiva del 'ahora': el alma debe pronunciar, por lo menos, dos 'ahora' sucesivos para que lo que queda determinado como intermedio entre ellos aparezca como un lapso o segmento temporal (cf. IV 11, 219a25-30), el cual luego se empleará como unidad de medida para medir el todo en cuestión. Según esto, la actividad numerante del alma está presupuesta no sólo en la posible determinación numérica de un tiempo total, sino también en la previa determinación de las unidades temporales que han de ser empleadas en la operación de cuenta o medida. En tal sentido, puede decirse que la propia numerabilidad del tiempo — y no sólo su efectivo 'haber sido numerado'- queda, como tal, en dependencia de la intervención activa del alma, al menos, en la medida en que ésta es la única instancia capaz de llevar a cabo la determinación de las unidades requeridas para el acto de numeración.

b) Un segundo aspecto importante viene dado por el hecho de que Aristóteles no afirma aquí que sólo haya tiempo cuando el alma realiza o ha realizado efectivamente la acción de numerar. La afirmación de Aristóteles es más modesta, pues establece tan sólo una conexión entre la existencia del alma y la numerabilidad en general, como lo muestra la formulación alternativa que alude a lo numerado o bien numerable. Aristóteles parece querer decir simplemente: para poder hablar de tiempo no basta la mera existencia de determinados procesos o movimientos, sino que se requiere, además, que dichos procesos o movimientos, en sí mismos indiferentes de toda determinación de su medida, sean puestos en conexión con una instancia capaz de llevar a cabo el acto de determinación correspondiente a través de la acción de numerar o contar. Dicho de otro modo: es en el ámbito de la relación movimiento-alma donde se abre, por primera vez, la posibilidad de la existencia del tiempo, en tanto número o medida de los procesos, y no en la esfera de los procesos o movimientos naturales como tales. ¿Qué queda entonces del tiempo, hecha abstracción de la presencia del alma en tanto agente de numeración? Aristóteles responde de modo unívoco en las líneas finales del pasaje: queda tan sólo aquello que oficia de 'sustrato' del tiempo (cf. 223a27: 'aquello siendo lo cual es el tiempo'), en la medida en que es aquello sobre lo cual recae la acción de numeración por parte del alma y provee la condición de la aplicación sucesiva del 'ahora'. Este 'sustrato' no es otra cosa que lo 'anterior y posterior en el movimiento', es decir, la sucesión provista por el movimiento mismo (cf. 223a28). Considerada por sí misma, esta sucesión no constituye todavía tiempo, 
pues el tiempo sólo aparece allí donde lo 'anterior y posterior en el movimiento’ es considerado como numerable (cf. 223a29) ${ }^{6}$.

A modo de balance puede establecerse lo siguiente. Contra lo que pretendería una lectura radicalmente realista del pasaje, hay que admitir que Aristóteles establece aquí una conexión estructural entre tiempo y alma, que excluye la posibilidad de la existencia efectiva del tiempo con independencia del alma como instancia capaz de llevar a cabo la acción de numerar el movimiento: en la concepción aristotélica el número, en el sentido de lo numerado y/o lo numerable, presupone la referencia a aquella instancia capaz de llevar a cabo el acto de numeración. Esta constatación concuerda con el diseño general de la teoría del tiempo desarrollada por Aristóteles en los capítulos precedentes. Téngase en cuenta que ya en el planteo inicial de las dificultades referidas a la relación tiempo-movimiento la referencia al alma como instancia capaz del conocimiento y la percepción del tiempo aparece en el primer plano de la consideración (cf. IV 11, 218b21-219a10), al punto que puede decirse incluso que los argumentos de Aristóteles muestran aquí una clara tendencia a borrar toda demarcación nítida entre la cuestión referida a la existencia del tiempo como tal, por un lado, y la cuestión referida a las condiciones que hacen posible la percepción o experiencia del tiempo, por el otro.

Contra la posibilidad de que Aristóteles asuma la existencia de una cierta dependencia estructural del tiempo respecto del alma se suele alegar que tal tesis sería incompatible con la posición general de Aristóteles, según la cual la existencia de los objetos sensibles no dependería de ser conocidos o percibidos por el sujeto ${ }^{7}$. Pero este modo de formular el problema enturbia definitivamente el punto central, porque apela a un empleo equívoco de la noción de ‘objeto sensible’. Lo que está en juego aquí no es la supuesta dependencia de la existencia de un objeto respecto de alguna actividad del alma, pues para Aristóteles el tiempo no es, como tal, un objeto, sino una determinación de los procesos a los que puede estar sometido un objeto sustancial, concretamente, el número de su movimiento. Por otra parte, aun si se tratara de un objeto sensible en el sentido de algo

${ }^{6}$ Como observa Hussey (1983) p. 173 ad loc., la idea de una sucesión provista por el movimiento, que, a falta del alma como instancia numeradora, no constituiría todavía tiempo, parece tener antecedentes en algunas sugerencias de Platón con referencia al movimiento desordenado que caracteriza a la materia en el estado pre-cósmico del universo. Cf. Timeo 30a, 52d-53a, 69b. Para este aspecto de la concepción platónica en el Timeo, véase Vlastos (1939).

${ }^{7}$ En tales términos plantea el problema Goldschmidt, en el marco de su defensa de una interpretación radicalmente 'realista' del pasaje de Fís. IV 14, 223a21-29. Véase p. ej. Goldschmidt (1982), p. 114. 
existente de modo sustancial e independiente, tampoco se trataría de la cuestión de su existencia o no como un objeto o cosa subsistente, sino, más bien, de las condiciones bajo las cuales dicho objeto puede desplegar efectivamente aquellas virtualidades que lo caracterizan en cuanto objeto sensible.

Puestas las cosas en estos términos, se advierte en seguida que la posición de Aristóteles en el caso del tiempo no constituye sino una aplicación específica de su concepción general en torno al estatuto de los objetos sensibles en cuanto sensibles. En efecto, la posición habitual de Aristóteles consiste justamente en que los objetos primarios de percepción (v. gr. colores, sabores, etc.) no tienen existencia actual independiente del acto mismo de la percepción, mientras que sus respectivos sustratos, es decir, los objetos materiales — que se denominan 'sensibles' sólo en sentido derivativo - pueden existir efectivamente con independencia de la actividad perceptiva como tal ${ }^{8}$. Algo análogo es lo que sostiene Aristóteles en el caso del tiempo: como número del movimiento, el tiempo presupone, para existir efectiva o actualmente, la referencia al alma como instancia capaz de llevar a cabo el acto de numeración, mientras que el sustrato del tiempo - es decir, la sucesión provista por el movimiento- puede existir incluso con independencia de dicha referencia.

Por otro lado, y contra lo que sería una lectura de corte idealista del texto, hay que llamar la atención sobre el hecho de que Aristóteles no afirma aquí en ningún momento ni que el tiempo esté en el alma misma, ni que sea, sin más, constituido espontáneamente por ella ${ }^{9}$. Aristóteles afirma tan sólo que el tiempo no existe efectivamente como tal sin el alma. El tiempo no es, para Aristóteles, ni una mera estructura subjetiva 'preformada' en el alma, ni un mero ens rationis carente de fundamento real. Para dar cuenta de la particular conexión estructural entre el tiempo y el alma que Aristóteles tiene a la vista, resulta muy acertada la caracterización de F. Volpi, cuando declara que el alma no posee aquí ni una función tético-constructiva, ni una función meramente constatativa, sino, más bien, una función de carácter constitutivo-manifestativa ${ }^{10}$. Aquí la noción de constitución debe entenderse en el sentido fenomenológico y no idealista, que hace caer el acento fundamentalmente en la función posibilitante que cumple la intervención de la actividad del alma para la manifestación de aquello que aparece y se muestra en sí mismo a través de dicha actividad. En tal sentido, puede decirse que la concepción aristotélica no se deja encasillar, sin más, bajo la

${ }^{8}$ Para este punto véanse Cat. 7, 7b33-8a6; Met. IV 5, 101b30-1011a2; De anima III 2, 426a15-26; Tóp. V 9, 138b30-37.

${ }^{9}$ Cf. Wieland (1970), p. 319.

${ }^{10}$ Cf. Volpi (1988), p. 58. 
alternativa habitual entre 'realismo' e 'idealismo', y ello, como veremos, ya por el hecho de que Aristóteles parte de una concepción de las relaciones entre el alma y el mundo que resulta esencialmente diferente de aquella que suele estar en la base de la polémica moderna en torno al problema de la relación entre el pensamiento y la realidad.

2) Uno de los aspectos que, a mi juicio, han perjudicado la interpretación del pasaje referido a la conexión entre tiempo y alma reside en el hecho de que incluso allí donde se ha dado con lecturas que hacen justicia en mayor medida a la posición fijada por Aristóteles, se ha tendido habitualmente a ver el pasaje como un argumento aislado y ocasional, que no revela una conexión estructural de fondo con el núcleo teórico de la física aristotélica. En alguna ocasión incluso se ha visto en el hecho de que Aristóteles no vuelva sobre el problema aquí planteado un indicio en favor de la suposición de que él mismo no otorgaba mayor relevancia a la cuestión o, al menos, a su propia respuesta al problema, que, por lo demás, sería lo suficientemente breve e insatisfactoria como para justificar tal actitud ${ }^{11}$. Pero, si bien es cierto que Aristóteles no vuelve expresamente a la cuestión planteada en IV 14, 223a21-29, no menos cierto es que la posición elaborada en el pasaje con referencia a la vinculación entre tiempo y alma guarda, desde el punto de vista estructural, una estrecha correspondencia con las posiciones que Aristóteles adopta en otros puntos centrales dentro de su concepción de la naturaleza y el movimiento natural. Ya me he referido al hecho de que el modo en que Aristóteles da cuenta de la conexión entre tiempo y alma debe verse, en definitiva, como una aplicación específica de su concepción general en torno al estatuto de los objetos sensibles en cuanto sensibles. Según Aristóteles, sólo en conexión con el acto perceptivo del alma los objetos sensibles primarios alcanzan existencia actual, mientras que, privados de tal conexión, quedan reducidos simplemente a sus correspondientes sustratos, que sólo son potencialmente sensibles, pero no pueden actualizar por sí mismos dicha potencialidad. Desde el punto de vista de la distinción categorial entre acto y potencia, Aristóteles expresa este punto clave en su concepción por recurso a la tesis que establece que el acto del sentido y el acto del correspondiente objeto sensible son uno y el mismo, aunque deban ser descriptos de modo diferente cuando se los considera desde el lado de lo que percibe y desde el lado de lo percibido, respectivamente (cf. De Anima III 2, 425b26). Dicho de otro modo: ocurre en estos casos que dos potencialidades diferentes, una situada del lado del 'sujeto' y otra situada del lado del 'objeto', para usar la terminología moderna habitual, comparten un único y mismo acto, en el cual convergen, de modo tal

${ }^{11}$ Así lo cree Ross (1936), p. 68. 
que cada una de ellas no puede llegar a su propia actualidad con independencia de la correspondiente actualización de la otra.

A esta estructura de complementariedad que caracteriza esencialmente a este tipo de actualización volveré más abajo. Por el momento baste con advertir que la posición fijada por referencia a dicha tesis de complementariedad resulta esencial dentro del modelo explicativo del movimiento natural que ofrece Aristóteles. Y bastaría en este respecto con que dicha tesis fuera imprescindible para dar cuenta del estatuto que corresponde a cualidades tales como los colores, los sabores o los sonidos, pues Aristóteles no opera con un modelo explicativo que reduzca las 'cualidades secundarias’, como se las llamó en la Modernidad, a las así llamadas cualidades primarias, que se corresponderían con las determinaciones puramente matemáticas de los cuerpos extensos. Por el contrario, las posteriormente así llamadas cualidades secundarias gozan para Aristóteles de un primado sistemático entre los objetos sensibles, en la medida en que constituyen los sensibles primarios o propios, en correspondencia con los diferentes órganos sensoriales. Y, por otra parte, en su concepción general del movimiento o cambio natural Aristóteles reconoce al cambio cualitativo un estatuto propio e irreductible, en la medida en que ubica a la alteración como una de sus cuatro especies básicas.

Con lo dicho bastaría para mostrar que la estructura de complementariedad avistada tanto en el tratamiento de la conexión tiempo-alma en Fís. IV 14 como en el tratamiento general del estatuto correspondiente a los objetos sensibles en cuanto sensibles resulta central dentro de la concepción de Aristóteles. Sin embargo, la Física ofrece también otros buenos ejemplos de aplicación del mismo tipo de modelo explicativo, los cuales conciernen, además, a aspectos centrales dentro de la filosofía natural aristotélica. El ejemplo más claro y sistemáticamente más relevante está dado, sin duda, por el tratamiento aristotélico del infinito en Fís. III 4-8. La noción de infinitud juega un papel central en la concepción de Aristóteles, en la medida en que se vincula de modo directo con la noción clave de continuidad. En efecto, Aristóteles trata la extensión espacial, el movimiento y el tiempo como tres formas o modalidades del continuum, que mantienen entre sí determinadas correlaciones estructurales. Tales correlaciones se fundan en correspondientes relaciones de prioridad y dependencia, en virtud de las cuales el tiempo depende en su existencia y sus propiedades del movimiento, y éste, a su vez, de la extensión espacial.

Ahora bien, el análisis del infinito que Aristóteles lleva a cabo en los capítulos mencionados presenta características muy peculiares, que le confieren un sorprendente aspecto de modernidad. Aristóteles desarrolla allí 
lo que con toda justicia puede denominarse una concepción fundamentalmente operacionalista del infinito, por oposición a las concepciones tendencialmente sustancialistas características de la tradición filosófica precedente. Las razones por las cuales Aristóteles debe necesariamente desplazarse en dirección de una concepción operacionalista, y no sustancialista, están conectadas con asunciones básicas de su ontología. Menciono las más importantes. En primer lugar, 'infinito' es una noción esencialmente predicativa, que, como tal, no designa de modo directo algo subsistente por sí mismo. Con la probable excepción de Anaximandro, esto fue advertido ya de algún modo, según Aristóteles, por la mayoría de los primeros filósofos naturales, los cuales no ponen como principio al infinito sin más, sino, más bien, a una naturaleza sustancial diferente ( $v$. gr. agua, aire o algún otro elemento), a la que atribuyen la infinitud como una determinación accidental (cf. III 4, 203a16 ss.). Radicalizando esta tendencia, apenas incoada en la posición de tales autores, Aristóteles rechaza expresamente la posibilidad de concebir el infinito en términos sustancialistas y enfatiza fuertemente su carácter esencialmente predicativo (véase esp. III 5, 204a8-34). Por otra parte, y en inmediata conexión con lo anterior, Aristóteles asume la imposibilidad de la existencia en el orden de la simultaneidad de cantidades infinitas, sean discretas o continuas. Por lo mismo, no puede haber, a su juicio, ni conjuntos de infinitos elementos coexistentes todos al mismo tiempo, ni tampoco cuerpos o magnitudes espaciales infinitamente extensas. Todo conjunto de elementos actualmente existentes tendrá una cantidad determinada de elementos, que, por grande que fuere, nunca será infinita (cf. III 5, 204b7-10). Y aun cuando la serie numérica pueda ser considerada como infinita, no hay en ella ningún número particular que sea él mismo infinito. Del mismo modo, toda extensión espacial tendrá una medida determinada y, con ello, límites, por lejos que éstos puedan estar situados (cf. III 5, 204b5-7; 204b10-206a9). Esto implica, para Aristóteles, que incluso el universo físico como un todo debe concebirse necesariamente como finito, desde el punto de vista de su extensión en el espacio (cf. p. ej. III 7, 207a15-18).

La concepción así elaborada queda resumida en la tesis —que, así formulada, no se encuentra en el texto, pero suele pasar por expresión genuina del núcleo de la posición aristotélica- según la cual no hay infinito en acto. Aquí la noción de acto o actualidad debe ser entendida en términos que involucran un componente esencialmente temporal, que remite al orden de la simultaneidad y la coexistencia: no hay ni conjuntos con infinito número de miembros que existan simultáneamente, ni hay magnitudes infinitamente extensas en el orden de la coexistencia provisto por el espacio. 
Sobre esta base, el desafío para Aristóteles consiste en la necesidad de dar cuenta de la posibilidad de la existencia de series infinitas en el orden de la sucesión, cuya generación pueda explicarse sin poner en cuestión el marco provisto por una concepción estrictamente finitista del universo físico en el orden de la simultaneidad y la coexistencia. En efecto, las razones que llevan a Aristóteles a reconocer la necesidad de conceder algún tipo de existencia al infinito son básicamente tres, y todas ellas se conectan de modo directo con la necesidad de hacer lugar a la posibilidad de series infinitas en el orden de la sucesión, a saber: por un lado, evitar tener que poner un principio o un fin a la sucesión temporal; por el otro, evitar poner límites a la divisibilidad de las magnitudes y abandonar así la presuposición de continuidad, al postular la existencia de magnitudes mínimas indivisibles; y, finalmente, evitar poner un límite arbitrario a la serie numérica (cf. III 6, 206a9-12). Como es sabido, Aristóteles resuelve el problema así planteado a través de la atribución al infinito de una existencia sólo potencial, en un sentido preciso de potencialidad, que excluye su actualización completa y que involucra, con ello, también un desplazamiento desde el orden de la simultaneidad y la coexistencia al orden de la sucesión: el caso del infinito no corresponde, por ejemplo, al de potencialidad propia del bronce de llegar a ser una estatua, la cual no excluye la posibilidad de su completa actualización, sino que corresponde, más bien, al modo de existencia de series sucesivas como el día o los juegos olímpicos, las cuales se dice que son o existen, en la medida en que en cada fase de la sucesión temporal se verifica la existencia de alguno de los miembros que las componen, pero sin que haya ningún momento en que se verifique la existencia simultánea de todos los miembros de la serie (cf. III 6, 206a18-25). La única y esencial diferencia del caso del infinito respecto de casos como el día y los juegos olímpicos consiste justamente en que los dos últimos son ejemplos de series sucesivas finitas, que poseen como tales un miembro que debe considerarse el último de la serie, mientras que el caso de las series infinitas excluye por principio la existencia de un miembro de la serie que pueda considerarse el último. Pero, independientemente de ello, ambos tipos de series sucesivas se caracterizan por el hecho de combinar inseparablemente en su estructura ontológica tanto un aspecto de actualidad y progresiva realización efectiva como un aspecto de potencialidad siempre abierta e inacabamiento, pues mientras la serie existe ocurre que, en cada fase de su existencia, existe(n) actual y efectivamente sólo alguno(s) de sus miembros, mientras que hay otros que ya no existen o bien aún no han llegado a existir, de modo que, en cuanto sucesiva, la serie, además de no existir nunca conjuntamente como un todo, queda en cada fase de su existencia siempre inacabada. En efecto, en el caso de una serie sucesiva finita, cuando con la venida a la existencia del último miembro la 
serie queda como tal concluida, entonces puede decirse que la serie misma como serie sucesiva justamente ya no existe.

En qué medida esta concepción, con su desplazamiento desde el orden de la simultaneidad y la coexistencia al orden de la sucesión, involucra al mismo tiempo un componente esencialmente operacionalista puede explicarse del modo más sencillo a partir del tratamiento aristotélico del así llamado ‘infinito por división', que guarda conexión directa con el problema de la continuidad. Aristóteles apunta aquí, ante todo, a mostrar que la posibilidad de dividir sin término una magnitud no requiere que ésta deba concebirse como infinitamente extensa, sino que se funda en las propiedades estructurales de cualquier magnitud extensiva, en cuanto toda magnitud de este tipo resulta divisible iterativamente en partes que presentan la misma naturaleza que el todo (cf. VI 2, $232 b 24$ s.; VI 8, 239a21 s.). Sobre esta base, explica Aristóteles, el proceso de división puede continuarse sin término, incluso allí donde se tome como punto de partida la magnitud extensiva más pequeña, con tal que la división se lleve a cabo según la regla de no dividir el total en partes iguales, sino más bien según una proporción constante del residuo obtenido en cada caso, por ejemplo, por la mitad de dicho residuo (cf. III 6, 206a33-b1; 206b12-16). Como lo reclama la posición finitista de base adoptada por Aristóteles en el plano correspondiente al orden de la simultaneidad y la coexistencia, la infinitud no aparece aquí como una propiedad efectivamente dada de la magnitud extensiva, sino, más bien, como una propiedad que ésta revela allí donde es sometida a determinado tipo de proceso de división, en la medida en que cada paso del proceso deja siempre abierta la posibilidad de reiterar la acción de división ejecutada en dicho paso. En cada paso del proceso de división, llevado a cabo según la regla de proporcionalidad prescripta al comienzo, la potencialidad que caracteriza a la magnitud como divisible es progresivamente actualizada y se pone de manifiesto como tal. Pero la posibilidad siempre abierta de continuar iterativamente el mismo proceso de actualización pone al mismo tiempo de manifiesto la imposibilidad de una actualización completa de dicha potencialidad, que la reduzca sin residuo a la pura efectividad. El componente esencialmente operacionalista de esta concepción de la infinitud se expresa de modo directo en el hecho de que el tipo de potencialidad que caracteriza ontológicamente al infinito sólo puede ponerse aquí de manifiesto a través de la mediación de determinadas operaciones, concretamente, de la operación de división, llevada a cabo según una cierta regla procedimental. La concepción de la infinitud así elaborada responde a los requerimientos de la posición finitista de base que Aristóteles adopta en el plano correspondiente al orden de la coexistencia y la simultaneidad, en la medida en que evita la 
introducción de conjuntos de infinitos elementos coexistentes o magnitudes espaciales infinitamente extensas. Pero, como se echa de ver, también la concepción operacionalista presupone la existencia de, al menos, una extensión infinita, concretamente, la del tiempo mismo, en la medida en que la posibilidad de iterar indefinidamente el proceso de división no puede hacerse plausible sino sobre el trasfondo que provee la representación de una sucesión temporal sin término. Sin embargo, esto no produce ninguna dificultad a la concepción aristotélica, sencillamente por la razón de que la serie sin término de las partes del tiempo está situada ya como tal en el orden de la pura sucesión, y no en el de la simultaneidad y la coexistencia.

Ahora bien, la conexión esencial que vincula infinitud y tiempo en la concepción aristotélica no se agota en el aspecto señalado, sino que afecta también al modo en que ambos se relacionan con el alma. En efecto, al igual que el tiempo, también la infinitud puede poner de manifiesto su propia actualidad sólo en conexión con el alma y a través de determinadas operaciones llevadas a cabo por ésta. Si el tiempo viene a la presencia efectiva, como tal, en correlación con el acto de numeración del alma, en el caso del infinito es el proceso de división el que permite poner de manifiesto su peculiar constitución ontológica, con la inseparable combinación de efectividad actualizada y potencialidad residual que la caracteriza. Apelando a la matriz explicativa provista por la tesis de la complementariedad de sentido y sensible en los procesos de actualización, puede resumirse el paralelo estructural entre el caso del tiempo y el del infinito, en lo que concierne a la relación con la actividad del alma, del siguiente modo: así como en el caso del tiempo el acto de la instancia capaz de numerar y el acto de lo numerable son uno y el mismo, también en el caso del infinito por división el acto de lo que tiene la facultad de dividir y el acto de lo que puede ser dividido sin término son uno y el mismo, pues es en y a través de la iteratividad del proceso de división como se pone de manifiesto la divisibilidad inagotable de aquello sobre lo cual recae el proceso, y sólo atendiendo a tal inagotabilidad residual podemos denominar al objeto en cuestión 'infinito'.

3) Recapitulando lo aportado por los dos casos discutidos puede decirse lo siguiente con relación a la caracterización de la perspectiva adoptada por Aristóteles en su abordaje temático de la naturaleza, en términos de una peculiar concepción indiferentista. Ambos tipos de actualidad, tanto el que caracteriza a la realidad propia del tiempo como el que caracteriza a la realidad propia del infinito, pertenecen esencialmente, en virtud de su propia estructura, a aquella dimensión de indiferenciación situada más allá o bien más acá de la distinción tajante entre el sujeto y el objeto del acto de conocimiento. Formulado de modo positivo: en ambos tipos de actualidad 
se expresa de modo peculiar la convergencia del alma y sus objetos en un mismo y único acto. Se trata aquí, puede decirse, de una experiencia unitaria que vincula inescindiblemente tanto el aporte originario del mundo como el aporte de la actividad del alma misma. Tanto en el caso del tiempo como en el del infinito, el contenido de dicha experiencia unitaria presenta un mismo tipo de estructura: por un lado, se tiene el continuum perceptible, sea la magnitud espacialmente extensa o bien el movimiento que un objeto lleva a cabo sobre ella; por otro, se tiene la intervención activa del alma, que aporta el momento de la discreción a través del acto de demarcación del correspondiente límite, sea por medio de la determinación del punto en el cual tiene lugar en cada caso la correspondiente división de la magnitud extensa, sea a través de la determinación del 'ahora' en el seguimiento del movimiento del móvil y en correlación con la sucesión de sus fases. En ambos casos, la intervención activa del alma, a través de la introducción del límite, eleva a una nueva forma de objetividad o presencia aquello que venía dado de manera inmediata a través de la simple captación pasiva en la percepción. Y lo hace a través de la actualización de potencialidades que están como tales ancladas en la propia estructura ontológica de aquello sobre lo cual recaen las correspondientes operaciones, pero que, en razón de su propia índole, no pueden alcanzar su propia actualización sino a través de la intervención activa de una instancia diferente.

He dicho al comienzo que el indiferentismo que caracteriza a la posición aristotélica, en tanto situada metódicamente en la dimensión de convergencia previa a toda separación tajante entre el ‘sujeto' y el ‘objeto' del conocimiento, está él mismo ontológicamente fundado. El alcance preciso de esto puede comprenderse ahora un poco mejor, a partir del peculiar carácter de complementariedad que caracteriza a los fenómenos de actualización avistados por Aristóteles. Tanto en el caso del tiempo como en el caso del infinito, el esquema de actualización subyacente presenta, como vimos, una misma estructura, que, metafóricamente hablando, podría denominarse de tipo triangular: las potencialidades propias de dos 'cosas' diferentes -en el caso del tiempo, el movimiento, en cuanto numerable, y el alma (o el noûs), en cuanto capaz de numerar; en el caso del infinito, la magnitud espacialmente extensa, en cuanto divisible, y el alma (o el noûs), en cuanto capaz de dividir - encuentran su correspondiente actualización en un mismo y único acto, en el cual ambas convergen y quedan elevadas a una nueva forma de objetividad o presencia ${ }^{12}$. Dicho acto puede ser des-

12 Para el noûs como instancia que aporta el momento de la discreción en el continuum perceptivo, y para las correlaciones noûs-límite y aísthesis-continuum, véanse también las excelentes observaciones de Wieland (1970) pp. 316 y ss., y 329 y ss. 
cripto de dos modos diferentes, según se adopte el punto de vista de una u otra entre las dos potencialidades intervinientes, a saber: el mismo acto puede ser descripto como acto de 'numerar'/'dividir', desde el punto de vista de la instancia activa, o de 'ser numerado'/‘ser dividido', desde el punto de vista de la instancia sobre la que recae la correspondiente operación. Pero se trata, sin embargo, de un mismo y único acto, en el cual ambas potencialidades convergen, y fuera del cual ninguna de ellas podría encontrar su propia actualización específica. En la esencial complementariedad que caracteriza a las estructuras triangulares de actualización reside, pues, en última instancia el fundamento ontológico de la decisión metódica por el indiferentismo, a la hora de tematizar la relación del alma con el mundo en el acto de conocimiento. El indiferentismo que caracteriza el abordaje fenomenológico practicado por Aristóteles en su tematización de la naturaleza está, por tanto, ontológicamente fundado, ya que la propia naturaleza sólo puede desplegar efectivamente todas sus potencialidades en conexión con las capacidades manifestativas del alma.

\section{Conclusión: alma, mundo, manifestación}

A modo de conclusión, retomo ahora muy brevemente algunos aspectos centrales y generalizo algunos de los resultados alcanzados en la discusión.

Como vimos, hay ciertas propiedades de los objetos de la realidad física que sólo vienen a su presencia efectiva y a su manifestación como tales con el concurso activo del alma. Esto no concierne tan sólo a propiedades como los colores, los sabores, los sonidos, etc., que en los enfoques de tipo causal-reduccionista, tanto de la Antigüedad como de la Modernidad, aparecen relegadas a un estatuto meramente secundario y epifenoménico. También vale, cambiando lo que hay que cambiar, para aquellas otras propiedades y estructuras fundamentales que - como la numerabilidad, la divisibilidad, la continuidad y el orden temporal — forman parte del entramado ontológico básico de la realidad física en su conjunto, es decir, de aquel ámbito del ser en el cual el fenómeno primario en el que adquiere expresión la constitución ontológica de las entidades básicas que lo componen no es otro que el fenómeno del movimiento y la procesualidad, en general. Esto muestra con suficiente claridad que no es posible hacer justicia a la concepción aristotélica si se proyecta acríticamente sobre ella el modelo explicativo basado en la habitual distinción entre las cualidades primarias y las cualidades secundarias. Se trata aquí de una distinción cuyos antecedentes 
más remotos se remontan, por lo menos, hasta Demócrito, pero que sólo a partir de la Modernidad se convirtió en el paradigma definitorio de un abordaje genuinamente científico de los fenómenos naturales, al ser asociado a la tesis ontológica y metódica de la prioridad de las determinaciones matemáticas, en el marco del proyecto general de matematización de la naturaleza. Aristóteles no suscribe dicha tesis, ni considera a las cualidades 'secundarias' como menos importantes o dotadas de menor respaldo objetivo que las cualidades y determinaciones puramente matemáticas. Y su concepción acerca de la esencial complementariedad de las estructuras de actualización subyacentes a todo acceso a la naturaleza tiende, más bien, a poner en un pie de igualdad ambos tipos de propiedades o determinaciones, en lo que respecta a su posibilidad de ser efectivamente actualizadas como tales.

Puede decirse, por tanto, que, a juicio de Aristóteles, el mundo abierto en la experiencia inmediata de la naturaleza no es, en su conjunto, tal como efectivamente se ofrece, sin el concurso activo del alma con su peculiar función manifestativa. Pero esto no debe entenderse simplemente en el sentido de la tesis —más o menos trivial y carente de ulteriores consecuencias - que afirma que, sin algo que percibe o conoce, nada es como tal percibido o conocido. Con su énfasis en la complementariedad esencial que caracteriza a las estructuras triangulares de actualización, Aristóteles va decididamente más allá de esta constatación elemental y poco vinculante, en la medida en que pone de relieve el papel indispensable que cumple la intervención activa del alma en la apertura de un nuevo ámbito de objetividad y manifestación, dentro del cual únicamente determinadas potencialidades de los objetos naturales pueden alcanzar la forma plena de efectividad que como tales les corresponde: sin la intervención activa del alma, el continuum en su diferentes formas - espacial, procesual, temporal- no se manifestaría como tal, pues no podría poner de manifiesto su potencialidad estructural de ser iterativamente divisible sin término; sin intervención activa del alma, el movimiento no pondría efectivamente de manifiesto su intrínseca numerabilidad y, con ello, tampoco el carácter esencialmente temporal de su sucesividad. El alma cumple un papel manifestativo ineliminable respecto de todos estos aspectos estructurales de la naturaleza. El famoso dictum aristotélico según el cual el alma es en cierto modo todas las cosas existentes (cf. De Anima III 8, 432b21 s.) revela, a la luz de estas conexiones, un alcance significativo que va mucho más allá de lo que pudiera sospecharse a primera vista.

Como ontología fenomenológica del ente sujeto a movimiento, la filosofía natural de Aristóteles no se orienta a partir de un ideal de objetivi- 
dad que exija una completa prescindencia del aporte procedente de la intervención activa del alma en la constitución del ámbito de experiencia que la propia filosofía natural pretende convertir en objeto de tematización. Por el contrario, la filosofía natural aristotélica se sitúa de antemano en el nivel descriptivo que corresponde a aquella dimensión de convergencia en la cual hay siempre ya manifestación, una dimensión en la cual la separación tajante entre lo que modernamente se denomina el ‘sujeto' y el 'objeto' de la correspondiente experiencia no encuentra ya ocasión de ser aplicada significativamente. Que esto está muy lejos de representar un punto de partida dogmático, que sanciona acríticamente la validez de la inmediatez, se comprende de inmediato cuando se advierte que la dimensión abierta en la experiencia inmediata de la naturaleza resulta como tal ineludible en cualquier discurso significativo sobre la realidad física en su conjunto. Esto vale también, y muy especialmente, para aquellos abordajes que se caracterizan por el intento de reducir por vía de explicación causal buena parte de los contenidos ofrecidos por dicha experiencia a instancias que se consideran más básicas o elementales. Pues cualquier intento causal-reductivo de este tipo sólo puede desplegar genuino potencial explicativo, en la medida en que en su ir más allá de lo inmediatamente dado no pierda definitivamente todo contacto con su propio punto de partida. En caso contrario, la pérdida definitiva de contacto con el explanandum haría al mismo tiempo superflua la apelación a aquellas instancias que han de cumplir la correspondiente función de explanans. Dicho de otro modo: ningún intento de abordaje de tipo causal-reductivo puede dejar definitivamente atrás la dimensión originaria de la experiencia inmediata, que es la que le provee no sólo su punto de partida fáctico, sino también, en definitiva, el punto final de llegada al que apuntan, de uno u otro modo, todos sus esfuerzos explicativos. Vistas las cosas desde esta perspectiva, es, más bien, el ideal de una supuesta objetividad no deudora de ningún punto de partida en la experiencia inmediata, y prescindente de todo aporte procedente de la intervención activa del alma en su función manifestativa, el que aparece como una pretensión dogmática e injustificada, derivada de la asunción acrítica de una peculiar manera de representarse las relaciones entre el alma y el mundo, que tiene ella misma un origen histórico identificable.

No es en absoluto casual que la posibilidad de una rehabilitación filosófica del modelo teórico provisto por la física aristotélica, acotado en sus pretensiones dentro de los límites que corresponden a su propio ámbito temático-descriptivo y a su diseño metódico específico, se haya ofrecido en una época como la nuestra, que ha debido presenciar drásticos cambios en los modelos explicativos fundamentales, tanto en el ámbito científico como 
en el propiamente filosófico. Se trata de cambios que han permitido una agudizada toma de conciencia de las presuposiciones ontológicas que trae siempre ya consigo todo intento de acceso temático a un determinado ámbito descriptivo. Tampoco es casual que el marco filosófico en el cual dicha rehabilitación ha quedado fácticamente inscripta haya sido el del auge de los enfoques que, tanto en el ámbito estrictamente filosófico ( $v$. gr. la fenomenología) como en el de la teoría de la ciencia ( $v$. gr. el constructivismo operacionalista y su derivación culturalista), apuntan a estrategias de fundamentación que buscan orientación a partir del mundo de la vida, como suelo fundamental de experiencia y horizonte último de sentido. Pues lo característico del enfoque aristotélico es su compromiso básico con el nivel descriptivo que corresponde al acceso inmediato a la naturaleza en la experiencia pre-reflexiva y pre-teórica. En dicho compromiso básico puede rastrearse la fuente originaria de la que emanan tanto su grandeza como también sus miserias.

\section{REFERENCIAS}

Allen, R. E. (ed.): Studies in Plato's Metaphysics. London: 1965.

Aubenque, P.: Le Problème de l'Être chez Aristote. Paris: cuarta edición, 1977 [1962].

Barnes, J., M. Schofield, R. Sorabji (eds.): Articles on Aristotle, Vol. 1, Science. London: 1975.

Bröcker, W.: Aristoteles. Frankfurt a. M.: cuarta edición, 1974.

Furley, D. J., R. E. Allen: Studies in Presocratic Philosophy, Vol. I., The Beginnings of Philosophy. London: 1970.

Goldschmidt, V.: Temps Physique et Temps Tragique chez Aristote. Paris: 1982.

Heidegger, M. (1939): "Vom Wesen und Begriff der Physis”. Citado por la reproducción en Heidegger, Wegmarken. Frankfurt a.: M. 1967 y reimpr., pp. 309-371.

Heidegger, M.: Wegmarken. Frankfurt a. M.: 1967 y reimpr.

Hussey, E.: Aristotle's Physics, Books III-IV. Oxford: 1983.

Popper, K.: "Back to the Presocratics”. En K. Popper, Conjectures and Refutations. London: 1963, cap. 5. Citado por la reproducción en Furley y Allen, Studies in Presocratic Philosophy, Vol. I., The Beginnings of Philosophy. London: 1970, pp. 130-153.

Ross, W. D.: Aristotle's Physics. Oxford: 1936 y reimpr.

Rudolph, E. (ed.): Zeit, Bewegung, Handlung. Studien zur Zeitabhandlung des Aristoteles. Stuttgart: 1988.

Vlastos, G.: "The Disorderly Motion in the Timaios". En Classical Quarterly, 33 (1939), pp. 71-83. Reproducido en Allen (ed.), Studies in Plato's Metaphysics. London: 1965, pp. 379-399.

Volpi, F.: "Chronos und Psyche. Die aristotelische Aporie von Physik IV, 14, 223 a 16-29”. En Rudolph (ed.), Zeit, Bewegung, Handlung. Studien zur Zeitabhandlung des Aristoteles. Stuttgart: 1988, pp. 26-62. 
Wieland, W.: "Das Problem der Prinzipienforschung und die aristotelische Physik”. En Kant-Studien, 52 (1960-61), pp. 206-219. Citado por la reproducción en Barnes-Schofield-Sorabji (eds.), Articles on Aristotle, Vol. 1, Science. London: 1975, pp. 127-140.

Wieland, W.: Die aristotelische Physik. Göttingen: 1970. Reimpresión corregida de la primera edición de 1962, con un nuevo "Nachwort". 